\title{
The Order of the Attaching Class in the Suspended Quaternionic Quasi-Projective Space
}

\author{
Dedicated to Professor Minoru Nakaoka on his 60th birthday
}

By

Juno MUKAI*

\section{$\S 0$. Introduction}

In this note, $F$ denotes the field of the complex numbers $C$ or the field of the quaternions $H$. We denote by $F P^{n}$ the $F$-projective space of $n F$-dimensions and by $Q_{n}(F)$ the quasi- $F$-projective space. $G_{n}(F)$ denotes the unitary group $U(n)$ or the symplectic group $S p(n)$ according as $F$ is $C$ or $H$. Let $d$ be the dimension of $F$ over the field of the real numbers $R$ and $S^{d n-1}$ the unit sphere in $F^{n}$. Let $T_{n}^{\prime}: S^{d n-2} \rightarrow G_{n-1}(F)$ be the characteristic map for the normal form of the principal $G_{n-1}(F)$-bundle over $S^{d n-1}$. Then, as is well known ([2]. [3] and [9]), $\operatorname{Im} T_{n}^{\prime}=Q_{n-1}(F)$, precisely, the following diagram commutes :

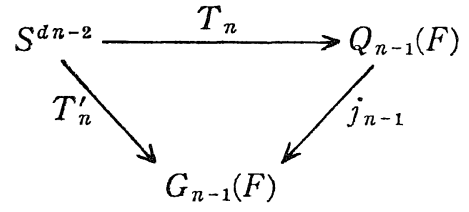

where $j_{n-1}$ is the canonical reflection map. $Q_{n}(F)=Q_{n-1}(F) \cup_{T_{n}} e^{d n-1}$ and $Q_{n}(C)$ $=E\left(C P_{+}^{n-1}\right)$, where $E(\quad)$ denotes the reduced suspension and $C P_{+}^{n-1}$ a disjoint union of $C P^{n-1}$ and \{one point .

Let $\omega_{n-1}=\omega_{n-1}(F)$ be the homotopy class of $T_{n}$ and $p: Q_{n}(C) \rightarrow Q_{n}(C) / Q_{1}(C)$ $=E C P^{n-1}$ the collapsing map. In the previous paper [6], we proved that the $k$-th suspension $E^{k}\left(p_{*} \omega_{n}(C)\right)$ is of order $n$ ! for $k \geqq 0$.

The purpose of this note is to examine the order of $E^{k} \omega_{n-1}(H)$.

Let $\alpha$ be an element of a homotopy group $\pi_{n}(\quad)$ and $E^{\infty} \alpha \in \pi_{n}{ }^{S}(\quad)$ the stable element of $\alpha . o(\beta)$ denotes the order of $\beta$. Then, our result is the following

Theorem. i) $o\left(E^{k} \omega_{n-1}(H)\right)=2 \cdot(2 n-1) !$ for $k \geqq 0$ if $n$ is even.

$$
\text { ii) } o\left(E^{\infty} \omega_{n-1}(H)\right)=(2 n-1) ! \quad \text { if } n \text { is odd. }
$$

Communicated by N. Shimada, June 10, 1983. Revised September 19, 1983.

* Department of Mathematics, Faculty of Liberal Arts, Shinshu University, Matsumoto 390 , Japan. 
Our method is essentially to use the $K$-theory. To examine $o\left(\omega_{n-1}(H)\right)$, we use Toda's theorem about the generator of $\pi_{2 n-1}(U(n))$ [6] and the group structure of $\pi_{t n+2}(S p(n))$ [4]. To determine the lower bound of $o\left(E^{k} \omega_{n-1}(H)\right)$, we use the standard method of D.M. Segal [7] from the unstable viewpoint, exactly, we use the Hurewicz homomorphism $h: \pi_{k+4 n-1}\left(E^{k} Q_{n}(H)\right) \rightarrow H_{k+4 n-1}\left(E^{k} Q_{n}(H) ; Z\right)$. A powerful tool is Toda-Kozima's map $\tilde{t}_{n}: Q_{n}(H) \rightarrow Q_{2 n}(C)$ [8].

In the stable case, our result overlaps with Corollary 4 of [5] and Theorem 5.8 of [9].

The author wishes to express his sincere gratitude to Professor Seiya Sasao for many advices given during the preparation of this paper.

\section{$\S 1$. Determination of $o\left(\omega_{n-1}(H)\right)$ for Even $n$}

First we recall the definition of the quasi-projective space and the reflection map. $S\left(F^{n}\right)$ denotes the unit sphere in $F^{n} . Q_{n}(F)$ is the space obtained from $S\left(F^{n}\right) \times S(F)$ by imposing the equivalence relation: $(u, q) \sim\left(u g, g^{-1} q g\right)$ for $g \in S(F)$ and collapsing $S\left(F^{n}\right) \times\{1\}$ to a point. The reflection map $j_{n}=j_{n}(F): Q_{n}(F) \rightarrow G_{n}(F)$ is defined as follows :

$$
j_{n}([u, q])(v)=v+u(q-1)\langle u, v\rangle
$$

for $u \in S\left(F^{n}\right), q \in S(F)$ and $v \in F^{n}$, where $\langle u, v\rangle=\sum_{k=1}^{n} \bar{u}_{k} v_{k}$ for $u=\left(u_{1}, \cdots, u_{n}\right)$ and $v=\left(v_{1}, \cdots, v_{n}\right)$.

Let $z=x+j y \in H$, where $x, y \in C$. By regarding $x \in C$ as $x+j 0 \in H$, we have the injection $C \subset \rightarrow H$. Obviously, this induces the canonical maps $i_{n}: Q_{n}(C) \rightarrow Q_{n}(H)$ and $i_{n}^{\prime}: U(n) \rightarrow S p(n)$. From the definition, the following diagram commutes:

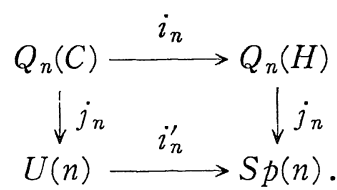

In the complex case, we can define the reduced reflection map [6]:

$$
\tilde{j}_{n}=\tilde{j}_{n}(C): E C P^{n-1} \cong Q_{n}(C) / Q_{1}(C) \longrightarrow U(n) / U(1) \cong S U(n) .
$$

By abuse of notation, we often use the same letter $j_{n}$ for the reduced case.

Lemma 1.2. i) If $n$ is even, $j_{n^{*}}: \pi_{4 n-1}\left(Q_{n}(H)\right) \rightarrow \pi_{i n-1}(S p(n))$ is an epimor phism. ii) If $n$ is odd, $\operatorname{Im} j_{n^{*}}=a \pi_{4 n-1}(S p(n))$, where $a=1$ or 2 .

Proof. Let $p: Q_{2 n}(C) \rightarrow Q_{2 n}(C) / Q_{1}(C) \cong E C P^{2 n-1}$ be the collapsing map, $k$ : $Q_{n}(H) \rightarrow Q_{2 n}(H)$ and $k^{\prime}: S p(n) \rightarrow S p(2 n)$ the inclusion maps, respectively. Then, by (1.1), the following diagram commutes for $r=4 n-1$ : 


$$
\begin{aligned}
& \pi_{r}\left(E C P^{2 n-1}\right) \stackrel{p_{*}}{\longleftarrow} \pi_{r}\left(Q_{2 n}(C)\right) \stackrel{i_{2 n^{*}}}{\longrightarrow} \pi_{r}\left(Q_{2 n}(H)\right) \stackrel{k_{*}}{\longleftarrow} \pi_{r}\left(Q_{n}(H)\right) \\
& \downarrow \tilde{j}_{2 n}(C)_{*} \quad \downarrow j_{2 n}(C)_{*} \quad \downarrow j_{2 n^{*}} \quad \downarrow j_{n^{*}} \\
& \pi_{r}(S U(2 n))=\pi_{r}(U(2 n)) \stackrel{i_{2 n^{*}}^{\prime}}{\longrightarrow} \pi_{r}(S p(2 n)) \stackrel{k_{*}^{\prime}}{\longleftarrow} \pi_{r}(S p(n)) .
\end{aligned}
$$

$p_{*}$ is an epimorphism since $Q_{2 n}(C) \simeq E C P^{2 n-1} \vee S^{1}$. By Theorem 4.1 of $[6]$, $\tilde{i}_{2 n}(C)_{*}$ is an epimorphism. So, $j_{2 n}(C)_{*}$ is an epimorphism. $k_{*}$ and $k_{*}^{\prime}$ are isomorphisms respectively. As is well known, $i_{2 n^{*}}^{\prime}$ is an isomorphism if $n$ is even and $\operatorname{Im} i_{2 n^{*}}^{\prime}=2 \pi_{n-1}(S p(2 n))$ if $n$ is odd. Therefore, the above commutative diagram leads us to the assertion. This completes the proof.

Proposition 1.3. i) $o\left(\omega_{n-1}\right)=2 \cdot(2 n-1)$ ! for even $n$.

ii) $o\left(\omega_{n-1}\right)=a \cdot(2 n-1)$ ! for odd $n$, where $a$ is the same number as in Lemma 1.2.

Proof. Let $p:\left(Q_{n}(H), Q_{n-1}(H)\right) \rightarrow\left(S^{1 n-1}, *\right)$ be the collapsing map. We consider the natural homomorphism between the exact sequences for $r=4 n-1$ :

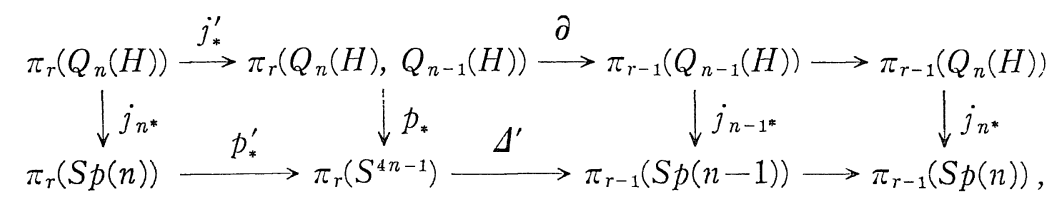

where the mappings are canonical and $\partial$ and $\Delta^{\prime}$ are the connecting homomorphisms.

As is well known, $\pi_{1 n-1}(S p(n)) \approx Z, \pi_{1 n-z}(S p(n)) \approx 0$ and $\pi_{m}\left(S^{m}\right)=\left\{\iota_{m}\right\} \approx Z$. By the Blakers-Massey theorem [1], $p_{*}$ is an isomorphism. By the definition, $\omega_{n-1}=\Delta\left(\iota_{4 n-1}\right)$, where $\Delta=\partial \circ p_{*}^{-1}$. So, by Theorem 2.2 of $[4], j_{n-1^{*}}$ is an epimorphism and the following holds:

$$
\begin{aligned}
& \pi_{1 n-2}(S p(n-1))=\left\{j_{n-1^{*}} \omega_{n-1}\right\} \approx Z_{b \cdot(2 n-1) !}, \text { where } b=1 \text { for odd } n \\
& \quad \text { and } b=2 \text { for even } n .
\end{aligned}
$$

By the exactness of the upper sequence, $o\left(\omega_{n-1}\right)$ is equal to the order of the cokernel of $j_{*}^{\prime}$. Hence, by (1.4), Lemma 1.2 and by the above commutative diagam, we have the assertion. This completes the proof.

By inspecting the above proof, we have the following

Proposition 1.5. $j_{n^{*}}: \pi_{ \pm n-1}\left(Q_{n}(H)\right) \rightarrow \pi_{1 n-1}(S p(n))$ is an epimorphism if and only' if $o\left(\omega_{n-1}\right)=b \cdot(2 n-1)$ !, where $b$ is the same number as in (1.4).

\section{$\S 2$. Some Fundamental Facts}

For $n \geqq 0, X_{n}$ denotes a connected finite CW-complex such that $X_{0}=\{*\}$ and $X_{n}=e^{0} \cup e^{r_{1}} \cup \cdots \cup e^{r_{n}}$ for $n \geqq 1$. Here $r=r_{n}=d n-\varepsilon$ with $\varepsilon=0$ or 1 and $d-\varepsilon \geqq 2$. 
$\theta_{n-1}: S^{r-1} \rightarrow X_{n-1}$ denotes the attaching map, and so $X_{n}=X_{n-1} \bigcup_{\theta_{n-1}} e^{r}$. For example, $X_{n}=F P^{n}(d=2$ or 4 and $\varepsilon=0)$ and $X_{n}=Q_{n}(H)(d=4$ and $\varepsilon=1)$.

Let $p: X_{n} \rightarrow X_{n} / X_{n-1}=S^{r}$ and $p^{\prime}:\left(X_{n}, X_{n-1}\right) \rightarrow\left(S^{r}, *\right)$ be the collapsing maps. Let $\partial: \pi_{r+m}\left(E^{m} X_{n}, E^{m} X_{n-1}\right) \rightarrow \pi_{r+m-1}\left(E^{m} X_{n-1}\right)$ be the connecting homomorphism. Then, $\left(E^{m} p^{\prime}\right)_{*}: \pi_{r+m}\left(E^{m} X_{n}, E^{m} X_{n-1}\right) \rightarrow \pi_{r+m}\left(S^{r+m}\right)$ is an isomorphism for $m \geqq 0$ [1], and we define a homomorphism $\Delta: \pi_{r+m}\left(S^{r+m}\right) \rightarrow \pi_{r+m-1}\left(E^{m} X_{n-1}\right)$ by the composition $\partial \circ\left(E^{m} p^{\prime}\right)_{*}^{-1}$. By the definition, $\Delta\left(\iota_{r+m}\right)=E^{m} \theta_{n-1}$, where the same letter is used for a mapping and its homotopy class.

Let $h=h_{m}: \pi_{r+m}\left(E^{m} X_{n}\right) \rightarrow H_{r+m}\left(E^{m} X_{n} ; Z\right) \approx Z$ for $m \geqq 0$ be the Hurewicz homomorphism and $h(n, m)$ the non-negative integer such that $\operatorname{Im} h=$ $h(n, m) H_{r+m}\left(E^{m} X_{n} ; Z\right)$. Then we have the following

Lemma 2.1. $o\left(E^{m} \theta_{n-1}\right)=h(n, m)$.

Proof. $j:\left(E^{m} X_{n}, *\right) \rightarrow\left(E^{m} X_{n}, E^{m} X_{n-1}\right)$ denotes the inclusion. Then, we consider the commutative diagram:

$$
\begin{aligned}
& \pi_{r+m}\left(E^{m} X_{n}\right) \stackrel{j_{*}}{\longrightarrow} \pi_{r+m}\left(E^{m} X_{n}, E^{m} X_{n-1}\right) \stackrel{\partial}{\longrightarrow} \pi_{r+m-1}\left(E^{m} X_{n-1}\right)
\end{aligned}
$$

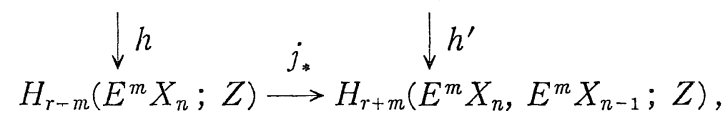

where $h^{\prime}$ denotes the relative Hurewicz homomorphism and the upper sequence is exact. From the cell structure of $X_{n}$, the lower $j_{*}$ is an isomorphism. By the relative Hurewicz theorem, $h^{\prime}$ is an isomorphism. This completes the proof.

According to [8], a representative element of $Q_{n}(H)$ can be taken as $\left(x+j y, e^{i \pi t}\right)$, where $x, y \in C^{n}$ satisfying $x+j y \in S\left(H^{n}\right)$ and $0 \leqq t \leqq 1$. Toda and Kozima defined $\tilde{t}_{n}: Q_{n}(H) \rightarrow Q_{2_{n}}(C)$ by the equation

$$
\tilde{t}_{n}\left[\left(x+j y, e^{i \pi t}\right)\right]=\left[\left(x \oplus y, e^{2 i \pi t}\right)\right] .
$$

We define $t_{n}: Q_{n}(H) \rightarrow E C P^{2 n-1}$ by the composition $p \circ \tilde{t}_{n}$, where $p: Q_{2_{n}}(C)$ $\rightarrow E C P^{2 n-1}$ is the collapsing map. From the definition. the following diagram commutes for $k<n$ :

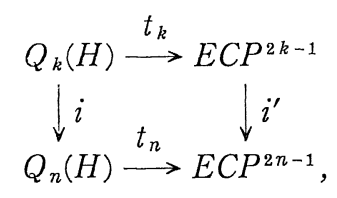

where $i$ and $i^{\prime}$ are the canonical inclusions.

The following lemma is a reduced version of Proposition 2.5 of [8].

Lemma 2.3 (Toda-Kozima). The following diagram commutes up to homotopy: 


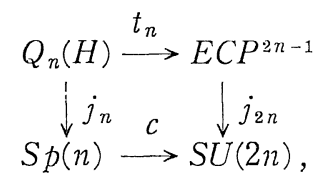

where $c$ is the complexification map.

Let $p: Q_{n}(H) \rightarrow Q_{n}(H) / Q_{n-1}(H)=S^{1 n-1}$ for $n \geqq 1$ and $p^{\prime}: E C P^{2 n-1} \rightarrow E C P^{2 n-1} /$ $E C P^{2 n-3} \simeq S^{4 n-3} \vee S^{1 n-1}$ for $n \geqq 2$ be the collapsing maps. Then, by (2.2), there exists a mapping $t_{n}^{\prime}: S^{1 n-1} \rightarrow S^{4 n-3} \vee S^{4 n-1}$ for $n \geqq 2$ such that the following diagram commutes:

$$
\begin{aligned}
Q_{n}(H) & \stackrel{t_{n}}{\longrightarrow} E C P^{2 n-1} \\
\underset{\downarrow}{\downarrow} p & \stackrel{t_{n}^{\prime}}{\downarrow} \operatorname{p}^{\prime} \\
S^{4 n-1} & \longrightarrow S^{1 n-3} \vee S^{1 n-1} .
\end{aligned}
$$

Let $p_{2}: S^{4 n-3} \vee S^{1 n-1} \rightarrow S^{1 n-1}$ for $n \geqq 2$ be the projection map. Then, we have the following

Lemma 2.5. $\operatorname{deg} t_{1}=-1$ and $\operatorname{deg}\left(p_{2} t_{n}^{\prime}\right)=(-1)^{n}$ for $n \geqq 2$.

Proof. We define $g_{n}: S\left(H^{n}\right) \rightarrow S\left(C^{2 n}\right)$ by the equation

$$
g_{n}(x+j y)=x \oplus y
$$

for $x, y \in C^{n}$. It is clear that $g_{n}$ is a homeomorphism and deg $g_{n}=(-1)^{n}$. By Lemma 2.3, $t_{1} \simeq g_{1}$ and $p_{2} t_{n}^{\prime} \simeq g_{n}$ for $n \geqq 2$. This completes the proof.

Hereafter the same letter is often used for a mapping and its homotopy class. Let $\gamma_{n}=\gamma_{n}(F): S\left(F^{n+1}\right) \rightarrow F P^{n}$ be the projection map. Let $i: E C P^{n-1} \rightarrow$ $E C P^{2 n}$ be the inclusion map. Then, we have the following

Proposition 2.6. $(-1)^{n+1} E \gamma_{2 n}(C)=i t_{n} \omega_{n}(H)$ 。

Proof. By (2.2) and (2.4), the following diagram commutes for $r=4 n+3$ :

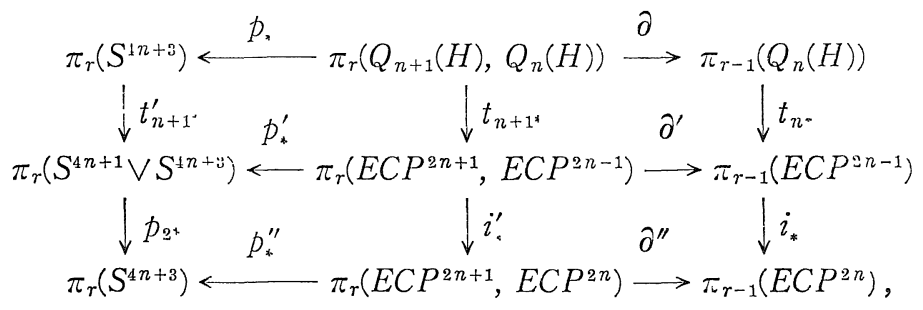

where the mappings are canonical.

$p_{*}$ and $p_{*}^{\prime \prime}$ are isomorphisms $[1]$. We note that $\omega_{n}(H)=\partial p_{*}^{-1}\left(\iota_{4 n+3}\right)$ and $E \gamma_{2 n}(C)$ $=\partial^{\prime \prime} p_{*}^{\prime \prime-1}\left(\iota_{4 n+3}\right)$. So, by Lemma 2.5 and the above commutative diagram, we have the assertion. This completes the proof. 
Remark 1. Owing to Proposition 2.6, it suffices to take $(-1)^{n+1} t_{n} \omega_{n}$ as $\lambda_{2 n}$ in Proposition 6.5. ii) of [6]. By Theorem 1.2 of [6] and Proposition 1.3, $o\left(\lambda_{2 n}\right)$ $=(2 n+1)$ ! or $2 \cdot(2 n+1) !$. In the last section, we shall show that $o\left(\lambda_{4}\right)=5$ ! icf. Lemma 11.1 of [6]).

\section{§3. Determination of the Lower Bound of $o\left(E^{m} \omega_{n-1}(H)\right)$}

Let $v \in \tilde{K}\left(C P^{2 n-1}\right)$ be the stable isomorphism class of the canonical line bundle over $C P^{2 n-1}$. We denote by $I_{C}: \tilde{K}(\quad) \rightarrow \tilde{K}\left(E^{2}\right)$ the Bott periodicity isomorphism. The following lemma is well known (cf. Lemma 2.2 of [8]).

Lemma 3.1. $I_{C}(v) \in \tilde{K}\left(E^{2} C P^{2 n-1}\right)$ is represented by the adjoint of the composite of the canonical maps:

$$
E C P^{2 n-1} \stackrel{j_{2 n}}{\longrightarrow} S U(2 n) \stackrel{i}{\longrightarrow} U(2 n) \stackrel{k}{\longrightarrow} \Omega B U(2 n),
$$

where $k$ is the homotopy equivalence.

Hereafter, $Z$ or the rational number field $Q$ is taken as the coefficients of the homology or cohomology groups, unless otherwise stated.

Let $\operatorname{ch}^{k}: \tilde{K}(\quad) \rightarrow H^{2 k}(\quad ; Q)$ be the $k$-th Chern character and $\operatorname{ch}=\sum_{k} \operatorname{ch}^{k}$ the total Chern character. Let $\sigma: \widetilde{H}^{i}(E) \rightarrow \widetilde{H}^{i-1}(\quad)$ be the suspension isomorphism. Then, as is well known, the following diagram commutes:

$$
\begin{aligned}
& \tilde{K}\left(C P^{2 n-1}\right) \stackrel{I_{C}}{\longrightarrow} \tilde{K}\left(E^{2} C P^{2 n-1}\right) \\
& \underset{H^{*}\left(C P^{2 n-1}\right.}{\downarrow} \mathrm{ch} \quad \stackrel{\sigma^{-2}}{\longrightarrow} \underset{\mathrm{l}}{\longrightarrow} H^{*}\left(E^{2} C P^{2 n-1} ; Q\right) .
\end{aligned}
$$

We denote by $y$ a generator of $H^{2}\left(C P^{2 n-1}\right)$. It is also well known that

$$
\operatorname{ch}^{2 n-1} v=1 /(2 n-1) ! y^{2 n-1} \text {. }
$$

Proposition 3.4. $o\left(E^{m} \omega_{n-1}\right)$ is a multiple of $(2 n-1)$ ! for $m \geqq 0$.

Proof. The assertion is a direct consequence of Theorem 1.2 of [6] and Proposition 2.6. For the later use, we give another proof for even $m$.

By (2.4) and Lemma 2.5, $t_{n}^{*}: H^{4 n-1}\left(E C P^{2 n-1}\right) \rightarrow H^{4 n-1}\left(Q_{n}(H)\right)$ is an isomorphism. So, $y^{\prime}=t_{n}^{*} \sigma^{-1} y^{2 n-1}$ is taken as a generator of $H^{4 n-1}\left(Q_{n}(H)\right)$. We choose a generator $x$ of $H_{4 n-1}\left(Q_{n}(H)\right)$ satisfying $\left\langle y^{\prime}, x\right\rangle=1$, where $\langle$,$\rangle denotes the$ Kronecker index.

Put $o\left(E^{m} \omega_{n-1}\right)=k(n)$. Denote by $s: \widetilde{H}_{\imath}(\quad) \rightarrow \widetilde{H}_{i+1}(E)$ the suspension isomorphism. Then, by Lemma 2.1, there exists an element $\alpha \in \pi_{m+4 n-1}\left(E^{m} Q_{n}(H)\right)$ satisfying $h_{m}(\alpha)=k(n) s^{m} x$. By the definition of the Hurewicz homomorphism, $h_{m}(\alpha)$ $=\alpha_{*} S^{n} \xi_{n}$, where $\xi_{n}$ denotes a generator of $H_{4 n-1}\left(S^{4 n-1}\right)$. So, we have $k(n)=$ 
$\left\langle\sigma^{-m} y^{\prime}, \alpha_{*} s^{m} \xi_{n}\right\rangle=\left\langle\alpha^{*} \sigma^{-m} y^{\prime}, s^{m} \xi_{n}\right\rangle$. Choose a generator $\tau_{n}$ of $H^{4 n-1}\left(S^{4 n-1}\right)$ satisfying $\left\langle\tau_{n}, \xi_{n}\right\rangle=1$. Then, we have $\alpha^{*} \sigma^{-m} y^{\prime}=k(n) \sigma^{-m} \tau_{n}$.

Put $m=2 t$ and $u=I_{C}{ }^{t}\left(E t_{n}\right) * I_{C}(v) \in \tilde{K}\left(E^{m+1} Q_{n}(H)\right)$. Then, by (3.2), (3.3) and by the naturality of the Chern character, we have the following:

$$
\sigma \operatorname{ch}^{2 n+t}(E \alpha)^{*} u=\alpha^{*} \sigma^{-m} t_{n}^{*} \sigma^{-1} \operatorname{ch}^{2 n-1}(v)=1 /(2 n-1) ! \alpha^{*} \sigma^{-m} y^{\prime} .
$$

So, we have $\operatorname{ch}^{2 n+t}(E \boldsymbol{\alpha})^{*} u=k(n) /(2 n-1) ! \sigma^{-m-1} \tau_{n}$. As is well known, Im $\mathrm{ch}^{2 n+t}$ $=H^{1 n+m}\left(S^{4 n+m} ; Z\right)$. Hence, $k(n) /(2 n-1) !$ is an integer. This completes the proof.

Lemma 3.5. $\left(E t_{n}\right)^{*} I_{C}(v)$ belongs to the image of the complexification homomorphism $c^{\prime}: \widetilde{K S p}\left(E Q_{n}(H)\right) \rightarrow \tilde{K}\left(E Q_{n}(H)\right)$.

Proof. By Lemmas 2.3 and 3.1, $u^{\prime}=\left(E t_{n}\right)^{*} I_{C}(v)=\left(\operatorname{adj}\left(k \circ i_{\circ} j_{2 n}(C)\right)\right)_{*}\left(E t_{n}\right)=$ $(\operatorname{adj} k)_{*}(E c)_{*}\left(E j_{n}(H)\right)$.

Let $\rho_{c}: B S p(n) \rightarrow B U(2 n)$ be the mapping induced from $c: S p(n) \rightarrow U(2 n)$ and $k^{\prime}: S p(n) \rightarrow \Omega B S p(n)$ be the canonical homotopy equivalence. Then, it is well known that $k^{\circ} c \simeq \Omega \rho_{c} \circ k^{\prime}$. So, we have $(\operatorname{adj} k)_{*}(E c)_{*}=\left(\rho_{c}\right)_{*}\left(\operatorname{adj} k^{\prime}\right)_{*}$. Hence, $u^{\prime}=\left(\rho_{c}\right)_{*}\left(\operatorname{adj} k^{\prime}\right)_{*}\left(E j_{n}(H)\right) \in \operatorname{Im} c^{\prime}$. This completes the proof.

As is well known, the following diagram commutes:

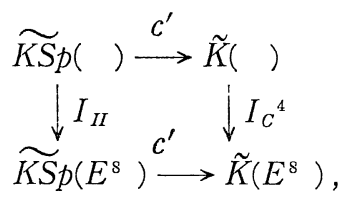

where $I_{I I}$ denotes the Bott periodicity isomorphism.

Proposition 3.7. If $n$ is even and $m \equiv 0 \bmod 8, o\left(E^{m} \omega_{n-1}\right)$ is a multiple of $2 \cdot(2 n-1) !$

Proof. As is well known, the following diagram commutes :

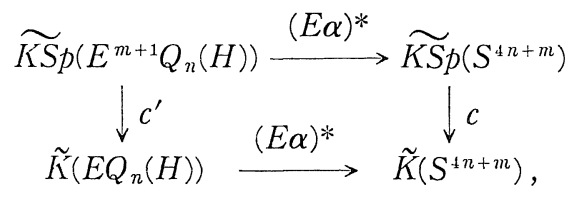

and $\operatorname{Im} c=2 \tilde{K}\left(S^{1 n+m}\right)$ if $n$ is even. So, by Lemma 3.5, (3.6) and by the proof of Proposition 3.4, $(E \alpha)^{*} u=(E \alpha)^{*} I_{C}{ }^{t}\left(E t_{n}\right) * I_{C}(v) \in 2 \tilde{K}\left(S^{4 n+m}\right)$ and $\mathrm{ch}^{2 n+t}(E \alpha) * u \in 2 H^{4 n+m}$ $\left(S^{4 n+m} ; Z\right)$. Therefore, $k(n) /(2 n-1)$ ! is an even integer. This completes the proof.

Remark 2. By the similar arguments, we have the following for $k \geqq 1$ (cf. [7]):

(1) $o\left(E^{k} \gamma_{n-1}(C)\right)$ is a multiple of $n$ ! for even $k$.

(2) $o\left(E^{k} \gamma_{n-1}(H)\right)$ is a multiple of $(2 n) ! / 2$ for even $k$. If $n$ is even and $k \equiv$ 
$0 \bmod 8, o\left(E^{k} \gamma_{n-1}(H)\right)$ is a multiple of $(2 n)$ !.

\section{$\S 4$. Proof of the Theorem}

To prove ii) of our theorem, we use the following [3]:

Theorem 4.1 (James). The stunted quasi-projective space $Q_{n}(F) / Q_{n-k}(F)$ is an S-retract of the factor space $G_{n}(F) / G_{n-k}(F)$ for $k \leqq n$. In particular, $j_{n^{*}}^{S}$ : $\pi_{\imath}^{S}\left(Q_{n}(H)\right) \rightarrow \pi_{\imath}^{S}(S p(n))$ is a monomorphism for $i \geqq 0$.

Now we are ready to prove the theorem. The assertion i) is a direct consequence of Propositions 1.3.i) and 3.7.

By Theorem 4.1, $j_{n-1^{*}}^{S}: \pi_{4 n-2}^{S}\left(Q_{n-1}(H)\right) \rightarrow \pi_{4 n-2}^{S}(S p(n-1))$ is a monomorphism. So, we have $o\left(E^{\infty} \omega_{n-1}\right)=o\left(E^{\infty} j_{n-1} \omega_{n-1}\right)$. Therefore, (1.4) and Proposition 3.4 lead us to the assertion. This completes the proof of the theorem.

Remark 3. We can give an improved proof of Theorem 1.2 of [6]. We use the first half of the proof of Theorem 1.2 of [6] and Remark 2.(1). We have

(1) $o\left(E^{k} \gamma_{n-1}(C)\right)=n$ ! for $k \geqq 1$.

By (1) and Remark 2.(2), we have the following:

(2) If $n$ is even, $o\left(E^{k} \gamma_{n-1}(H)\right)=(2 n)$ ! for $k \geqq 1$.

By Theorem 1.1 of [7] and by Lemma 2.1,

(3) $o\left(E^{\infty} \gamma_{n-1}(H)\right)=(2 n) ! / 2$ if $n$ is odd.

In this case, the Adams spectral sequence is used for the 2-primary stable homotopy of quaternionic and complex projective spaces [7].

\section{§5. An Example}

An open problem is to determine the order of $\omega_{n}(H)$ completely. The author hopes that an affirmative answer is given to the following

Conjecture. $o\left(\omega_{n-1}(H)\right)=(2 n-1)$ ! if $n$ is odd.

In this section, we determine the group structure of $\pi_{10}\left(Q_{2}(H)\right)$ and we show that the conjecture is true for $n=3$. We use the following: $\pi_{11}\left(S^{10}\right) \approx Z_{2}, \pi_{10}\left(S^{7}\right)$ $=\left\{\nu_{7}\right\} \approx Z_{24}, \pi_{11}\left(S^{7}\right) \approx 0, \pi_{9}\left(S^{3}\right) \approx Z_{3}$ and $\pi_{10}\left(S^{3}\right) \approx Z_{15}$.

Example. $\pi_{10}\left(Q_{2}(H)\right) \approx Z_{5 !} \oplus Z_{2}$ and $o\left(\omega_{2}(H)\right)=5$ !.

Proof. Let $p:\left(Q_{2}(H), S^{3}\right) \rightarrow\left(S^{7}, *\right)$ be the collapsing map. Then, $p_{*}$ : $\pi_{7}\left(Q_{2}(H), S^{3}\right) \rightarrow \pi_{7}\left(S^{7}\right)$ is an isomorphism [1]. We choose a generator $\alpha$ of $\pi_{7}\left(Q_{2}(H), S^{3}\right) \approx Z$ such that $p_{*} \alpha=\iota_{7}$.

$S p(2)$ is regarded as the cell complex $Q_{2}(H) \cup e^{\tau_{, 3}}$. Let $p^{\prime}:\left(S p(2), Q_{2}(H)\right) \rightarrow$ $\left(S^{10}, *\right)$ be the collapsing map. Then, $p_{*}^{\prime}: \pi_{n}\left(S p(2), Q_{2}(H)\right) \rightarrow \pi_{n}\left(S^{10}\right)$ is an isomorphism for $n \leqq 11$ [1]. 
We consider the following commutative diagram:

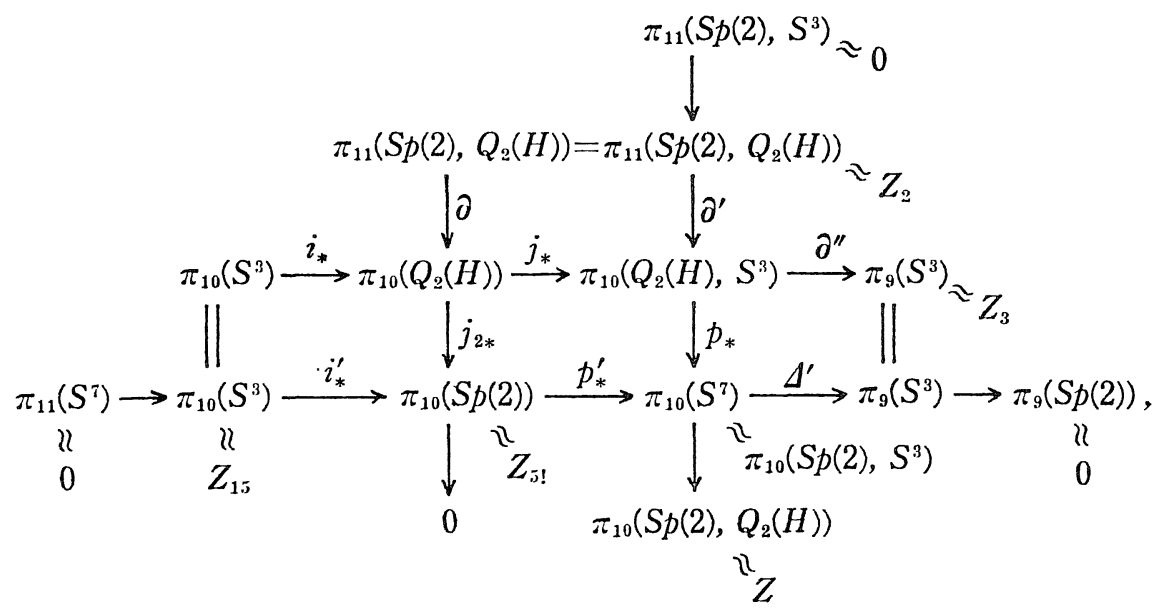

where the mappings are canonical and the horizontal and perpendicular sequences are exact respectively.

$p_{*}$ is a split epimorphism since $p_{*}\left(\alpha \nu_{7}\right)=\nu_{7}$. So, we have $\pi_{10}\left(Q_{2}(H), S^{3}\right) \approx$ $Z_{21} \oplus Z_{2}$. By the commutativity of the above diagram, $i_{*}$ is a monomorphism and $\partial^{\prime \prime}$ is an epimorphism. Therefore, by the upper horizontal sequence, $\pi_{10}\left(Q_{2}(H)\right)$ $\approx Z_{5 !} \oplus Z_{2}$. Hence, by Proposition 1.3. ii), we have $o\left(\omega_{2}\right)=5$ !. This completes the proof.

\section{References}

[1] Blakers, A.L. and Massey, W. S., The homotopy groups of a triad. II, Ann. of Math., 55 (1952), 192-201.

[2] James, I. M., Spaces associated with Stiefel manifolds, Proc. London Math. Soc,, (3) 9 (1959), 115-140.

[3] - The Topology of Stiefel Manifolds, London Math. Soc, Lecture Note, 24, Cambridge, 1976.

[4] Mimura, M. and Toda, H., Homotopy groups of symplectic groups, J. Math. Ky'oto Univ., 3 (1964), 251-273.

[5] Morisugi, K., Stable self maps of the quaternionic (quasi-) projective space, to appear.

[6] Mukai, J., The $S^{1}$-transfer map and homotopy groups of suspended complex projective spaces, Math. J. Okayama Univ., 24 (1982), 179-200.

[7] Segal, D. M., On the stable homotopy of quaternionic and complex projective spaces, Proc. Amer. Math. Soc., 25 (1970), 838-841.

[8] Toda, H. and Kozima, K., The symplectic Lazard ring, J. Math. Kyoto Univ., 22 (1982), 131-153.

[9] Walker, G., Estimates for the complex and quaternionic James numbers, Quart. J. Math. Oxford, (2) 32 (1981), 467-489. 
